\title{
Early cytotoxic effects of ochratoxin A in rat liver: A morphological, biochemical and molecular study
}

\author{
Nicoletta Gagliano $^{\mathrm{a}, *}$, Isabella Dalle Donne ${ }^{\mathrm{b}}$, Carlo Torri ${ }^{\mathrm{a}}$, Massimiliano Migliori ${ }^{\mathrm{c}}$, \\ Fabio Grizzi $^{\mathrm{d}}$, Aldo Milzani ${ }^{\mathrm{b}}$, Cristina Filippi ${ }^{\mathrm{c}}$, Giorgio Annoni ${ }^{\mathrm{e}}$, \\ Piergiuseppe Colombo ${ }^{\mathrm{f}}$, Francesco Costa ${ }^{\mathrm{a}}$, Giorgia Ceva-Grimaldi ${ }^{\mathrm{d}}$, \\ Alberto A.E. Bertelli ${ }^{a}$, Luca Giovannini ${ }^{c}$, Magda Gioia ${ }^{a}$ \\ ${ }^{a}$ Department of Human Morphology-LITA Segrate, Via Fratelli Cervi 93, 20090 Segrate, Milan, Italy \\ ${ }^{\mathrm{b}}$ Department of Biology, University of Milan, Milan, Italy \\ ${ }^{\mathrm{c}}$ Department of Neuroscience (Pharmacology Section) and Internal Medicine, University of Pisa, Italy \\ ${ }^{\mathrm{d}}$ Laboratories of Quantitative Medicine, Istituto Clinico Humanitas IRCCS, Rozzano, Milan, Italy \\ ${ }^{\mathrm{e}}$ Department of Clinical Medicine, Prevention and Medical Biotechnology and S. Gerardo Hospital, \\ University of Milano-Bicocca, Milan, Italy \\ ${ }^{\mathrm{f}}$ Department of Pathology, Istituto Clinico Humanitas IRCCS, Rozzano, Milan, Italy \\ Received 9 May 2006; received in revised form 1 June 2006; accepted 6 June 2006 \\ Available online 10 June 2006
}

\begin{abstract}
We characterized the overall early effect of chronic ochratoxin A (OTA) treatment on rat liver, analyzing different aspects related to: (i) fibrosis, by measuring collagen content and turnover, and $\alpha$-smooth muscle actin ( $\alpha$ SMA); (ii) oxidative stress and stress response, by analyzing protein carbonylation, superoxide dismutase (SOD) and heat shock protein (HSP70) gene expression; (iii) the possible tumor promoter effect, evaluating cadherin and connexin (CX) mRNA levels. Light microscopy analysis showed no histological differences in OTA-treated and control (CT) rats. Collagen content, determined by computer analysis of Sirius redstained liver sections, was similar in both groups. In liver homogenates COL-I, COL-III, TIMP-1 and TGF- $\beta 1$ mRNA levels and $\alpha$ SMA were unaffected by OTA. Matrix metalloproteinase (MMP)-1, MMP-2 and MMP-9 protein levels were also similar in the two groups. Protein carbonylation, a marker of severe oxidative stress, was not evident in the homogenates of OTA-treated livers; superoxide dismutase (SOD) mRNA tended to be lower and HSP70 was strongly down-regulated. OTA reduced E-cadherin and DSC-2 transcription, and down-regulated liver CX26, CX32 and CX43. In conclusion, these in vivo results show that OTA-induced liver injury involves a reduction in the ability to counterbalance oxidative stress, maybe leading to altered gap junction intercellular communication and loss of cell adhesion and polarity. This suggests that mild oxidative damage might be a key factor, in combination with other cytotoxic effects, in triggering the promotion of liver tumors after exposure to OTA.
\end{abstract}

(C) 2006 Elsevier Ireland Ltd. All rights reserved.

Keywords: Ochratoxin A; Collagen turnover; Oxidative stress; Cadherins; Connexins; HSP70

\footnotetext{
* Corresponding author. Tel.: +3902 50330462; fax: +390250330452 .

E-mail address: nicoletta.gagliano@unimi.it (N. Gagliano).
}

\section{Introduction}

The mycotoxin ochratoxin A (OTA) is produced by some species of fungi such as Aspergillus and Penicillum, as a secondary metabolite during storage of food. 
It is thus a widespread contaminant of a variety of animal and human foodstuffs, including cereal and grain products, coffee, beer and wine (O'Brien and Dietrich, 2005 for review). Contamination of food samples with OTA and the identification of OTA in blood of animals and humans living in endemic regions have been frequently described after consumption of contaminated food (Petkova-Bocharova et al., 1988; Li et al., 2000).

OTA exerts several toxic effects, mainly involving the kidney and liver. It is also a strong carcinogen in rats and mice (Petzinger and Ziegler, 2000), with immunosuppressive, teratogenic and genotoxic activity, and affects blood coagulation and carbohydrate metabolism (PfohlLeszkowicz et al., 2002). Epidemiological studies have provided evidence of a correlation between high OTA levels in blood and foods, and the development of Balkan endemic nephropathy, a chronic renal disease causing progressive fibrosis and impaired function, where extensive contamination with OTA is described (PetkovaBocharova et al., 1988; Vrabcheva et al., 2004).

The metabolic pathway of OTA and its effects vary from one tissue to another and although OTA-induced nephrotoxicity has been widely described, analysis of the overall effects of OTA on liver is still scant. To assess the human health risk posed by OTA the molecular events it triggers in the liver must be known. We therefore used morphological, molecular and biochemical methods to investigate the overall early alterations underlying OTA hepatotoxicity experimentally in an in vivo model. Our first goal was to see whether OTA induced fibrosis like in the kidney, so we investigated liver histology and the expression of genes and proteins involved in the collagen turnover pathway. Second, since OTA is reported to promote epithelial-to-mesenchymal transition (EMT) in kidney, we checked whether the toxin also modified the epithelial hepatocyte phenotype, particularly the expression of cadherins. Since OTA acts as a carcinogen, we analyzed whether it triggered carcinogenicity in the liver by epigenetic mechanisms, modifying the hepatocyte gap junction apparatus and influencing the expression of specific markers such as alpha-fetoprotein (AFP). Last, it has been reported that OTA toxicity is mediated by oxidative pathways (Baudrimont et al., 1994), so we analyzed protein carbonylation in liver homogenates, as a marker of severe oxidative stress damage, and the stress response induced in liver cells by chronic exposure to OTA.

\section{Materials and methods}

\subsection{Animals}

Sixteen male Wistar rats weighing 180-200 g and housed in single metabolic cages with controlled temperature $\left(25^{\circ} \mathrm{C}\right)$ and a 12-h alternating light-dark cycle were fed standard chow and had free access to drinking water. All animals received humane care and experiments were conducted in accordance with local ethical committee guidelines.

Rats were divided into two groups of eight, as follows:

- Group 1 (CT): untreated controls. Each rat was given $0.5 \mathrm{~mL}$ of saline by gastric gavage.

- Group 2 (OTA): OTA-treated rats. Rats were given a constant dose of OTA (Sigma-Aldrich), $289 \mu \mathrm{g} / \mathrm{kg}$ body weight every other day for 90 days by gastric gavage, as previously reported (Baudrimont et al., 1994). OTA was dissolved in $0.1 \mathrm{M} \mathrm{NaHCO}, \mathrm{pH} 7.4$, then diluted in $0.5 \mathrm{~mL}$ saline.

Finally, after 90 days the animals were weighed, euthanased by an anesthetic overdose (chloral hydrate), and the livers were removed. Liver fragments were processed for morphological examination or immediately frozen and stored at $-80^{\circ} \mathrm{C}$ for molecular analysis.

\subsection{Clinical chemistry}

Aspartate aminotransferase (AST), alanine aminotransferase (ALT), lactate dehydrogenase (LDH) and albumin (ALB) in plasma were assayed using standard protocols, according to the manufacturers' instructions.

\subsection{Histochemistry and image analysis}

Immediately after removal, liver fragments were fixed in $4 \%$ formalin in $0.1 \mathrm{M}$ phosphate-buffered saline (PBS), $\mathrm{pH}$ 7.4 , routinely dehydrated and paraffin-embedded. Serial sections ( $5 \mu \mathrm{m}$ thick) interspaced at $100 \mu \mathrm{m}$ were stained with hematoxylin-eosin, for routine histopathology, or Sirius red. For Sirius red staining, slides were de-paraffined and immersed for $30 \mathrm{~min}$ in saturated aqueous picric acid containing $0.1 \%$ Sirius red F3BA (Sigma, Milan, Italy), which specifically stains COL proteins distinctly red. Five sections were examined for each rat liver.

All the Sirius red-stained sections were analyzed by light microscopy and the images were captured and digitalized using an image analysis system with specific software (Isole, ICH, Italy). This software automatically selects the collagenous portion on the basis of similarities in the color of adjacent pixels, based on an RGB system. Tissue COL content is expressed by a fibrosis index (\%) that indicates the mean Sirius red-stained area as a percentage of the mean whole area of the section, calculated as the mean of the fibrosis indexes for each section for each rat.

\section{4. $R T-P C R$}

Total RNA was extracted from approximately $100 \mathrm{mg}$ of frozen liver by a modification of the acid guanidinium thiocyanate-phenol-chloroform method (Tri-Reagent, Sigma, Milan, Italy). RNA purity and concentration were determined 
Table 1

Primers sequences and RT-PCR amplification conditions

\begin{tabular}{|c|c|c|c|c|}
\hline Gene & Primer sequence & $\begin{array}{l}\text { Annealing } \\
\text { temperature }\left({ }^{\circ} \mathrm{C}\right)\end{array}$ & No. of cycles & bp \\
\hline$C O L-I$ & 5'-TTGACCCTAACCAAGGATGC 5'-CACCCCTTCTGCGTTGTATT & 54 & 30 & 197 \\
\hline COL-III & 5'-AGGCCAATGGCAATGTAAAG 5'-GGCCTTGCGTGTTTGATATT & 53 & 32 & 187 \\
\hline$T G F-\beta 1$ & 5'-CACCTGCACAGCTCCAGGCAC, 5'-CTTGCGACCCACGTAGTAGACG & 53 & 32 & 153 \\
\hline$T I M P-1$ & 5'-TCCCCAGAAATCATCGAGAC, 5' -ATGGCTGAACAGGGAAACAC & 55 & 32 & 329 \\
\hline$A F P$ & 5'-TGGAGAAGTGCTCCCAGTCT, 5'-GCAGTGGTTGATACCGGAGT & 57 & 32 & 359 \\
\hline$C X 26$ & 5'-TTAAAA CGTTGGCCT TTTGG, 5'-ACCCCATCTTGTTTCTGCAC & 55 & 30 & 378 \\
\hline$C X 32$ & 5'-AATGAGGCAGGATGAACTGG, 5' -CCTCAAGCCGTAGCATTTTC & 55 & 28 & 339 \\
\hline$C X 43$ & 5'-GCTCCACTCTCGCCTATGTC, 5'-TAGGTGCATGTTCTGCAAGC & 55 & 30 & 390 \\
\hline E-cadherin & 5'-GCAGTTCTGCCAGAGAAACC, 5' -AATCCTGCTTCCAGGGAGAT & 55 & 28 & 315 \\
\hline$N$-cadherin & 5'-TGTTGCTGCAGAAAACCAAG, 5'-GGCGACTCTCTGTCCAGAAC & 53 & 28 & 309 \\
\hline$D S G-2$ & 5'-CCTTGGTGGACCAGCACTAT, 5'-GACAGCACCGTCTTCCTAGC & 57 & 30 & 316 \\
\hline$D S C-2$ & 5'-CGTAATTCCCGTCCGAGTTA, 5'-TTGAGCTGTGAAGCCATTTG & 55 & 30 & 386 \\
\hline HSP70 & 5'-CGCATCCCCCAAGGTGCAGAA, 5'-CCAAGCACCTTCTTCTTGGTCCA & 55 & 32 & 691 \\
\hline$S O D$ & 5'-CGTCATTCACTTCGAGCAGA, 5'-CACCTTTGCCCA AGTCATCT & 55 & 28 & 341 \\
\hline$G A P D H$ & 5'-ATGGTGAAGGTCGGTGTGAAC, 5'-GCTGACAATCTTGAGGGAGT & 54 & 28 & 473 \\
\hline
\end{tabular}

spectrophotometrically. One microgram of total RNA was DNase I digested then reverse transcribed in $20 \mu \mathrm{L}$ final volume of reaction mix (Promega Italia, Milan, Italy).

The primers and protocols for RT-PCR are listed in Table 1. Amplification reactions were conducted in a final volume of $25 \mu \mathrm{L}$ containing $2.5 \mu \mathrm{L}$ of cDNA, $200 \mu \mathrm{M}$ of the four dNTPs, $100 \mathrm{pmol}$ of each primer, and $2.5 \mathrm{U}$ of Taq DNA polymerase (EuroTaq, Euroclone, Pero, Milan, Italy). Table 2 lists the RTPCR protocols. The RT-PCR products were electrophoresed on $1.5 \%$ agarose gels, stained with ethidium bromide and quantified by densitometric analysis (Image Pro-Plus). The results were normalized on GAPDH gene expression.

\subsection{Protein analysis}

Liver samples were homogenized in ice-cold extraction buffer (1 mL/100 mg tissue) containing Tris- $\mathrm{HCl} 50 \mathrm{mM} \mathrm{pH}$ 7.5, $\mathrm{NaCl} 100 \mathrm{mM}, \mathrm{CaCl}_{2} 2 \mathrm{mM}$. The homogenates were centrifuged $\left(4^{\circ} \mathrm{C}, 5 \mathrm{~min}, 14,000 \times g\right)$, and the supernatant was decanted and saved on ice. The final concentration of the liver extracts was determined with a standardized colorimetric assay (DC Protein Assay, Bio-Rad, Segrate, Milan, Italy), and the samples were divided into aliquots and stored at $-20^{\circ} \mathrm{C}$.

\subsubsection{Western blot}

For each sample $40 \mu \mathrm{g}$ of total protein extracts were diluted in SDS-sample buffer, loaded on $10 \%$ SDS-polyacrylamide

Table 2

Mean body weight (BW) and wet liver weight (LW), and ratio of LW to BW in CT and OTA-treated rats

\begin{tabular}{llll}
\hline Treatment & BW $(\mathrm{g})$ & LW $(\mathrm{g})$ & LW:BW ratio \\
\hline CT & $9.63 \pm 1.12$ & $413 \pm 20.4$ & $0.023 \pm 0.003$ \\
OTA & $9.56 \pm 1.72$ & $355 \pm 31.8$ & $0.027 \pm 0.006$ \\
\hline
\end{tabular}

Data are mean \pm S.D. gel, separated under reducing and denaturing conditions at $80 \mathrm{~V}$ according to Laemmli, and transferred at $90 \mathrm{~V}$ to a nitrocellulose membrane in $0.025 \mathrm{M}$ Tris, $192 \mathrm{mM}$ glycine, $20 \%$ methanol, $\mathrm{pH}$ 8.3. After electroblotting, the membranes were air-dried and blocked for $1 \mathrm{~h}$, washed in TBST (TBS/Tween-20 $0.05 \%$ ) and incubated with the specific antibodies.

To measure matrix metalloproteinase (MMP)-1 and -2 the membrane reacted with monoclonal antibodies to MMP-1 or MMP-2 $\left(1 \mu \mathrm{g} / \mathrm{mL}\right.$ in PBST/BSA $1 \% / \mathrm{NaN}_{3} 0.02 \%$, Calbiochem, San Diego, CA, USA) overnight at $4{ }^{\circ} \mathrm{C}$, and with an HRP-conjugated rabbit anti-mouse serum (1:80,000 dilution, Sigma, Milan, Italy); the immunoreactive bands were revealed with an amplified Opti-4CN (Bio-Rad, Segrate, Milan, Italy) and scanned densitometrically.

To assay $\alpha$ SMA, membranes were incubated for $1 \mathrm{~h}$ at room temperature in monoclonal antibody to $\alpha$ SMA (1:500 in TBST: Clone 1A4, Sigma, Milan, Italy) and in HRP-conjugated rabbit anti-mouse serum (1:80,000 dilution, Sigma, Milan, Italy). Immunoreactive bands were revealed using Bio-Rad Opti$4 \mathrm{CN}$.

\subsubsection{SDS-zymography}

Liver extracts were thawed on ice and mixed 3:1 with substrate gel sample buffer (10\% SDS, $4 \%$ sucrose, $0.25 \mathrm{M}$ Tris- $\mathrm{HCl} \mathrm{pH} 6.8,0.1 \%$ bromophenol blue). Each sample $(30 \mu \mathrm{g})$ was loaded under non-reducing conditions onto electrophoretic mini-gels (SDS-PAGE) containing $1 \mathrm{mg} / \mathrm{mL}$ of type I gelatin (Sigma, Milan, Italy). The gels were run at a running buffer temperature of $4{ }^{\circ} \mathrm{C}$.

After SDS-PAGE the gels were washed twice in $2.5 \%$ Triton X-100 for $30 \mathrm{~min}$ each, rinsed in water and incubated overnight in a substrate buffer at $37^{\circ} \mathrm{C}$ (Tris- $\mathrm{HCl} 50 \mathrm{mM}$, $\mathrm{CaCl}_{2} 5 \mathrm{mM}, \mathrm{NaN}_{3} 0.02 \%, \mathrm{pH} 8$ ). The gels were stained with Coomassie brilliant blue R250, and MMP gelatinolytic activity was detected as clear bands on a blue background. To confirm the identity of this activity, purified MMP-1 and 
MMP-2 (100 ng, Calbiochem, San Diego, USA) were run as standards.

\subsubsection{Protein carbonyl detection by Western blotting}

Anti-dinitrophenyl-KLH antibodies and horseradish peroxidase-conjugated goat anti-rabbit IgG, were purchased from Molecular Probes (Eugene, OR, USA). The Opti-4CN Substrate Kit and Precision-Plus Protein standards, molecular weights ranging from 10 to $250 \mathrm{kDa}$, were purchased from Bio-Rad Laboratories (Hercules, CA, USA). All other reagents were of analytical grade.

Protein samples $(30 \mu \mathrm{g})$ were mixed with $2 \times$ SDS sample buffer, then examined by SDS-PAGE using Criterion Tris- $\mathrm{HCl}$ $12.5 \%$ resolving gels (Bio-Rad Laboratories, USA). Gels were stained with Coomassie Brilliant Blue R-250 to verify the equal loading of samples. Samples were blotted at $100 \mathrm{~V}$ to a polyvinylidene difluoride membrane followed by derivatization with 2,4-dinitrophenylhydrazine (DNPH, $0.1 \mathrm{mg} / \mathrm{mL}$ in $2 \mathrm{~N} \mathrm{HCl})(10)$. After washing and membrane blocking as previously described (Dalle-Donne et al., 2002), protein carbonylation was evaluated immunologically for $2 \mathrm{~h}$ with antiDNP-KLH antibody $[1: 10,000$ dilution in $5 \%(\mathrm{w} / \mathrm{v})$ dried, non-fat milk in PBST: $10 \mathrm{mM}$ Na-phosphate, $\mathrm{pH} 7.2,0.9 \%$ (w/v) $\mathrm{NaCl}, 0.1 \%(\mathrm{v} / \mathrm{v})$ Tween 20] followed by 1-h incubation with horseradish peroxidase-conjugated goat anti-rabbit IgG (1:2000 dilution in 5\% (w/v) dried, non-fat milk in PBST) (Dalle-Donne et al., 2002). Immunoreactive bands were developed with the Opti-4CN Substrate Kit.

\subsection{Statistical analysis}

Results are given as mean \pm S.E.M. Experimental groups were compared by $t$-test. A $p$ value $<0.05$ was considered significant.

\section{Results}

\subsection{Liver and body weights and liver function}

The liver weights (LW) and body weight (BW) of CT and OTA-treated rats are presented in Table 2. Neither treatment had any effect on liver weight, and the ratio of LW to BW was similar in all groups. Serum ALT and LDH tended to be higher after OTA. ALB and AST were respectively lowered and raised in OTA-treated rats $(p<0.05$ versus CT) (Table 3$)$.
Table 4

Effect of OTA on fibrosis-related gene expression

\begin{tabular}{lllll}
\hline Treatment & COL-I & COL-III & TIMP-1 & TGF- $\beta 1$ \\
\hline CT & $0.939 \pm 0.06$ & $1.18 \pm 0.22$ & $1.24 \pm 0.06$ & $1.04 \pm 0.05$ \\
OTA & $0.828 \pm 0.08$ & $1.22 \pm 0.35$ & $1.07 \pm 0.06$ & $0.95 \pm 0.07$
\end{tabular}

Data are expressed as normalized densitometric units and are mean \pm S.D.

\subsection{Morphological and quantitative image analysis}

Histopathological analysis of hematoxylin and eosinstained rat liver sections indicated that liver morphology was the same in OTA-treated rats and CT, although in some OTA-treated livers a mild steatosis was detectable. Light microscopy of Sirius red-stained paraffin-embedded liver sections indicated a similar COL distribution in CT and OTA-treated rats. Accordingly, COL content, expressed as the fibrosis index, was not affected $(0.86 \pm 0.23$ and $0.86 \pm 0.12$, respectively, in CT and OTA-treated rats).

\subsection{Expression of fibrosis-related genes and proteins in liver}

COL-I and COL-III gene expressions were similar in CT and OTA-treated rats, as also TIMP- 1 and mRNA TGF- $\beta 1$ mRNA levels (Table 4).

MMP-1 and MMP-2 fell slightly after OTA (Figs. 1a, 2a, and 2b), while MMP-9 tended to be higher (Figs. $1 \mathrm{~b}$ and 2c). $\alpha$ SMA was similarly expressed in the two groups (Fig. 2d).

\subsection{OTA-induced oxidative stress damage and stress response}

Protein carbonyl formation, indicative of severe protein oxidation, was assayed in liver homogenates by Western blotting, probed with anti-DNP antibody after carbonyl derivatization with DNPH. OTA did not increase the protein carbonyl content. However, it strongly down-regulated HSP70 mRNA levels (74\%, $p<0.05$ versus CT) (Fig. 3a). SOD also tended to be lower $(18 \%, p=0.072)$ (Fig. 3b).

Table 3

Effect of OTA on clinical chemical parameters in rat blood

\begin{tabular}{lllll}
\hline Treatment & Albumin (U/L) & AST (U/L) & ALT (U/L) & LDH (U/L) \\
\hline CT & $3.413 \pm 0.25$ & $148.3 \pm 61.4$ & $39.5 \pm 16.4$ & $1365.5 \pm 435.7$ \\
OTA & $2.736 \pm 0.42^{*}$ & $208.0 \pm 142.1$ & $78.7 \pm 52.1^{*}$ & $2340.8 \pm 1806.0$ \\
\hline
\end{tabular}

Data are mean \pm S.D.

${ }^{*} p<0.05$ vs. CT. 


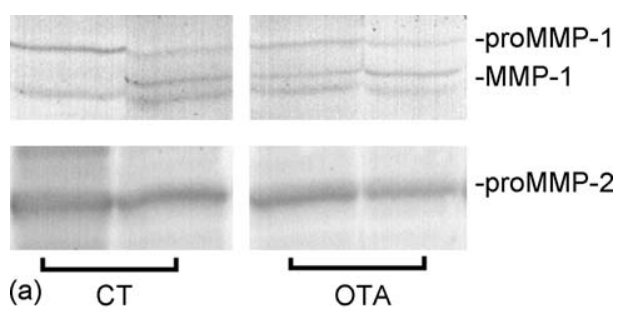

(a)

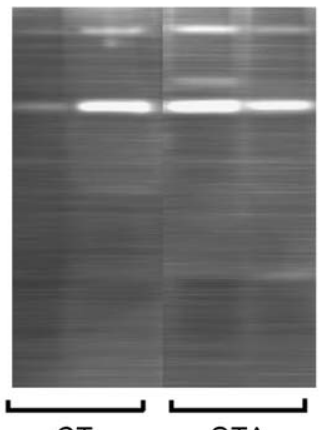

(b)

$$
\text { CT OTA }
$$

Fig. 1. (a) Western blot analysis for proMMP-1 and MMP-2. For MMP-1, the antibody recognized positive bands in the 52/57 and $42 / 46 \mathrm{kDa}$ regions corresponding respectively to the proenzyme and the active forms of MMP-1. (b) Representative SDS-zymography showing a lysis band consistent with proMMP-9. Each lane represents a single rat.

\subsection{Liver connexin and cadherin gene expression}

In liver homogenates CX26, CX32 and CX43 gene expressions fell steeply after OTA (by respectively
HSP70 mRNA

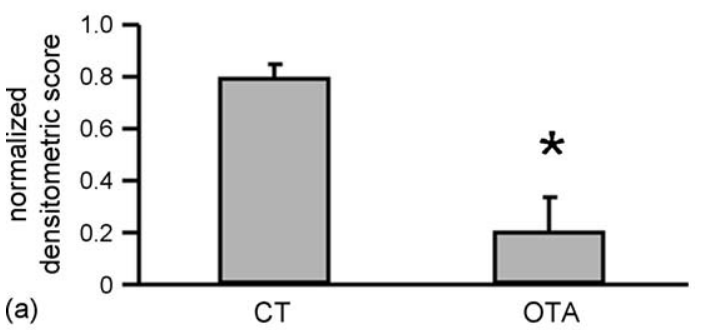

SOD mRNA

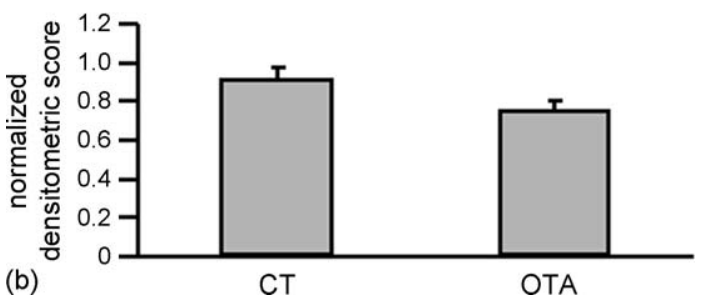

Fig. 3. Bar graphs showing HSP70 (a) and SOD (b) mRNA levels in liver homogenates of $\mathrm{CT}$ and OTA-treated rats. Changes in mRNA are expressed as normalized densitometric units relative to GAPDH mRNA. Means \pm S.E.M. $* p<0.05$ vs. CT.

$17 \%, 22 \%$ and $43 \%, p<0.05$ versus CT) (Fig. $4 a-c)$. The relative expression of the three connexins was not affected (Fig. 4d). OTA had no effect on DSG-2 mRNA levels. By contrast, DSC-2 gene expression was strongly down-regulated $(23 \%, p<0.05$ versus
MMP-1

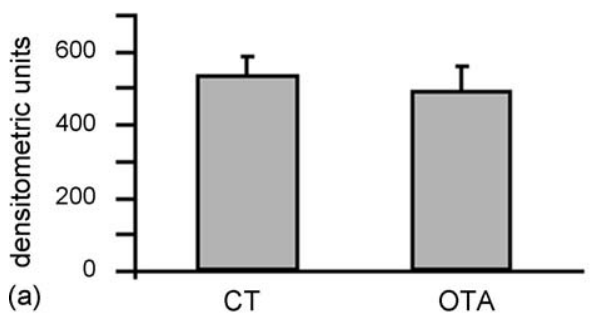

proMMP-9

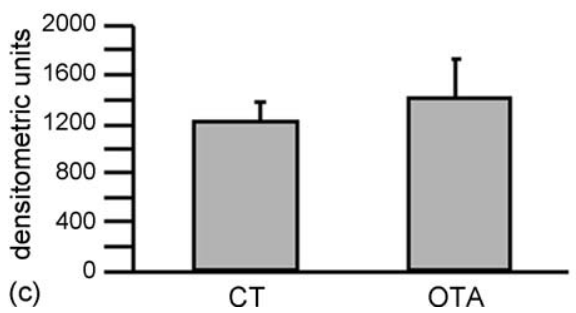

proMMP-2

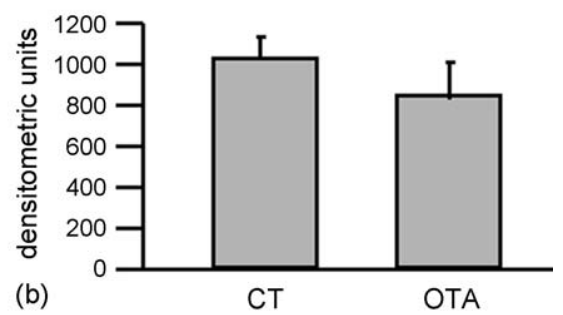

$\alpha \mathrm{SMA}$

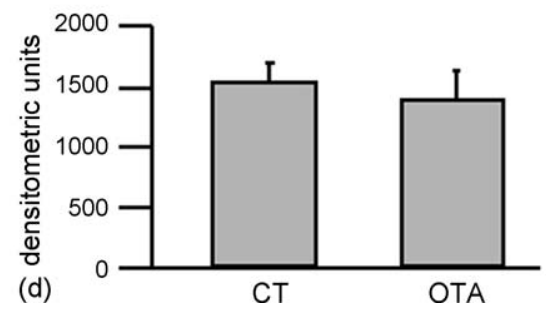

Fig. 2. Bar graphs showing MMP-1 (a), proMMP-2 (b), proMMP-9 (c) and $\alpha$ SMA (d) protein levels in CT and OTA-treated rats after densitometric analysis of immunoreactive bands. Means \pm S.E.M. of duplicate experiments. 
CX26 mRNA

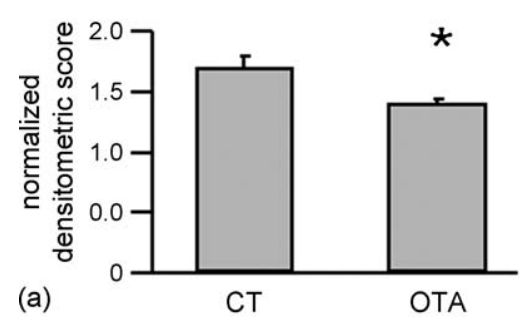

CX43 mRNA

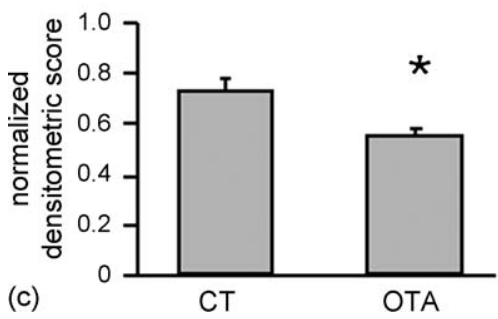

CX32 mRNA

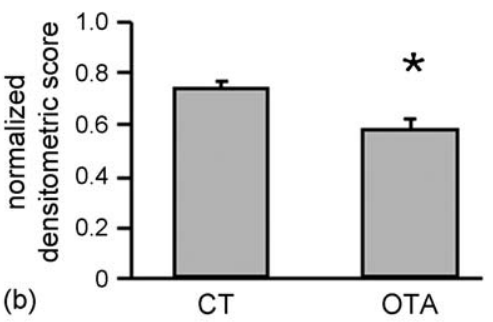

$\%$ CX expression

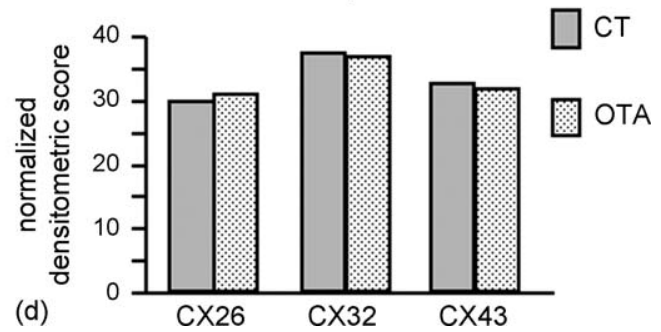

Fig. 4. Bar graphs showing CX26 (a), CX32 (b) and CX43 (c) mRNA levels in liver homogenates of CT and OTA-treated rats. Changes in mRNA are expressed as normalized densitometric units relative to GAPDH mRNA. (d) Relative expression of liver connexins. Means \pm S.E.M. $* p<0.05$ vs. CT.

CT) (Fig. 5a and b). N-cadherin tended to be reduced $(17 \%, \mathrm{NS})$, and E-cadherin mRNA levels were also lowered by OTA $(26 \%, p<0.05$ versus CT) (Fig. 5c and d).

\subsection{AFP $m R N A$ levels}

OTA increased AFP gene expression by $20.7 \%$ ( $p=$ $0.082)$ compared to $\mathrm{CT}(1.34 \pm 0.11$ versus $1.11 \pm 0.05)$.
DSC-2 mRNA

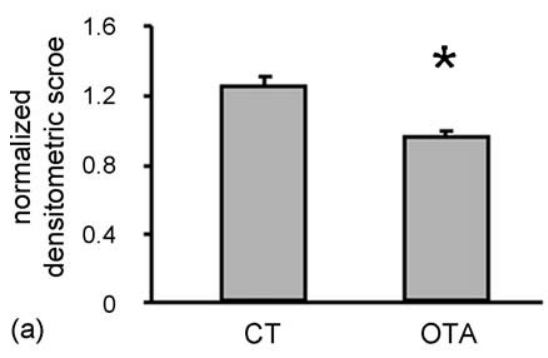

E-cad mRNA

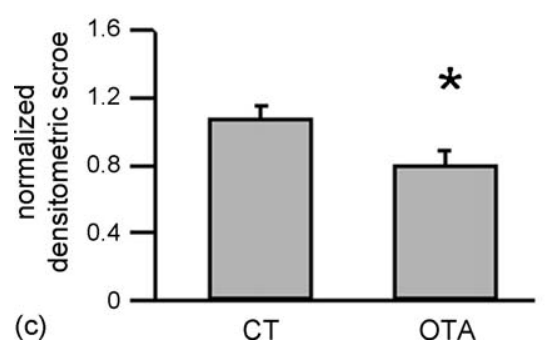

DSG-2 mRNA

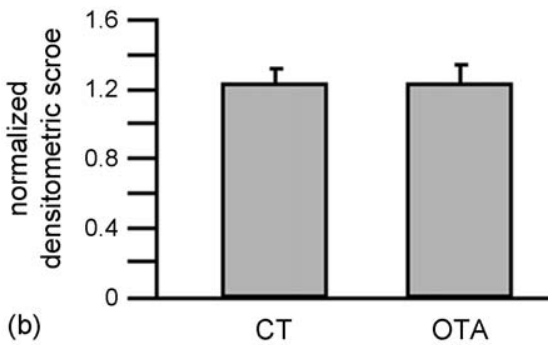

N-cad mRNA

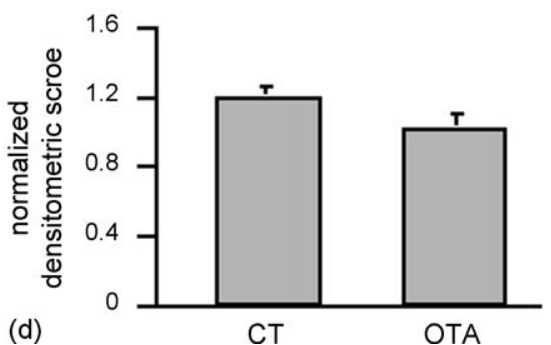

Fig. 5. Bar graphs showing DSG-2 (a), DSC-2 (b), E-cadherin (c) and N-cadherin (d) gene expression in CT and OTA-treated rats. Changes in mRNA are expressed as normalized densitometric units relative to GAPDH mRNA. Means \pm S.E.M. * $p<0.05$ vs. CT. 


\section{Discussion}

\subsection{Liver morphology and function}

Descriptions of OTA-induced liver histological modifications vary. In line with others (Kamp et al., 2005a; Mally et al., 2005a), we found no major morphological changes in the livers of OTA-treated rats compared to CT. The presence of a mild steatosis in OTA-treated rats, although with the absence of other liver lesions, is consistent with plasma AST and ALT. (Aydin et al. 2003), however, reported granular and vacuolated degeneration, cell necrosis, sinusoidal and central vein dilatation. These differences are not the result of different regimens. In fact, in both studies OTA was given either daily or on alternate days. Therefore, the possibility that more frequent repeated oral doses of OTA possibly caused a dosedependent rise in the OTA concentration in plasma and liver, inducing more evident histopathological lesions, cannot explain these results, and further studies will be necessary.

\subsection{Effect of OTA on collagen turnover}

Light microscopy of Sirius red-stained liver sections indicated a similar collagen content in CT and OTAtreated rats, suggesting that the toxin did not trigger a fibrotic process, unlike in the renal cortex (Gagliano et al., 2005). However, since collagen turnover is a highly tuned dynamic process, we examined whether OTA affected the balance between COL synthesis and degradation. Our data, showing a similar expression of genes and proteins involved in the synthesis and degradation pathways of COL, and of its modulation, suggest that OTA did not affect collagen turnover at the transcriptional, transductional and post-transductional levels. These findings enlight that the molecular mechanisms underlying OTA hepatotoxicity are different in the liver and the kidney, and that fibrosis is not a histopathological lesion typical of OTA-induced liver injury. This is supported by the similar $\alpha$ SMA expression in both experimental groups, indicative of a lack of activated myofibroblasts in the liver of OTA-treated rats (Cassiman et al., 2002).

\subsection{Oxidative damage and heat shock response}

Oxidative stress is the term generally used to describe a condition in which cellular antioxidant defenses are inadequate to completely inactivate the reactive oxygen species (ROS) generated. On account of excessive ROS production, loss of antioxidant defenses or both. Endoge- nous antioxidant defenses include glutathione (GSH), antioxidant vitamins and enzymes, such as SOD, catalase (CAT), glutathione reductase (GR) and peroxidase (GPx), as well as proteins involved in inducible cellular defense mechanisms such as heat shock proteins (HSP).

When ROS generation exceeds the rate at which endogenous antioxidants can scavenge it, or when endogenous antioxidant defenses are reduced or weakened, macromolecules become targets for oxidative modifications. Oxidative damage can severely compromise cell homeostasis and viability, or induce a variety of cellular responses through generation of secondary reactive species, ultimately leading to cell death. The effects of oxidative stress can be gleaned from the analysis of biomarkers of oxidative stress/damage, including oxidatively modified DNA bases, lipid peroxidation end-products, and oxidized proteins (Dalle-Donne et al., 2006).

When eaten in food, OTA passes into the blood and accumulates in organs such as the kidney and liver where, according to several reports, it increases ROS production (Schaaf et al., 2002). In rats repeated exposure to OTA resulted in high plasma and organ concentrations (increasing dose dependently). In the kidneys there were also unusual changes in renal histopathology, but in the liver there appeared to be no toxicity (Mally et al., 2005a). Consistent with the lack of effects on lipid peroxidation and the increase in DNA oxidative damage, these findings suggested that overt oxidative stress leading to tissue necrosis is unlikely to account for the potent toxicity of OTA.

An increase in malondialdehyde (MDA), a biomarker of lipid peroxidation, was reported in serum and in the liver and kidney of OTA-treated rats (Meki and Hussein, 2001; Ozcelik et al., 2004). MDA levels were measured as thiobarbituric acid reactivity (TBARS) by spectrophotometric assay. Although this technique is easy to use, the TBARS test is of questionable validity (Halliwell and Whiteman, 2004). Therefore, although the TBARS assay is accepted as an indicator of oxidative stress, it quantifies MDA-like material and does not specifically measure MDA or lipid peroxidation (Dalle-Donne et al., 2006). Actually, MDA measured by gas chromatography-mass spectrometry did not show any increase in rat plasma, kidney and liver (Gautier et al., 2001).

OTA was reported to increase 8-oxo-7,8-dihydro2 'deoxyguanosine $(8-\mathrm{OH}-\mathrm{dG})$ formation in rat liver and kidney and in various cell lines (Schaaf et al., 2002; Kamp et al., 2005a). However, in rats a single dose of OTA did not induce 8-OH-dG formation in kidney DNA (Gautier et al., 2001). 
Protein carbonylation is a sign of irreversible oxidative damage, often leading to a loss of protein function, which may have lasting detrimental effects on cells and tissues (Dalle-Donne et al., 2003a). The formation of protein carbonyls is currently the most widely used marker of severe oxidative stress, and a number of assays have been developed for carbonyl quantification (DalleDonne et al., 2003b). We found no increase in protein carbonylation in the liver of OTA-treated rats, in agreement with observations in kidney and liver (Kamp et al., 2005a; Mally et al., 2005a).

Since non-specific oxidative damage is often observed during toxicity, it is difficult from the analysis of MDA, 8-OH-dG, and protein carbonyls - presumed late biomarkers of oxidative stress/damage - to establish whether severe oxidative stress is the cause or the consequence of OTA-induced cell toxicity. Levels of early markers of oxidative stress, including GSH and its oxidized forms, antioxidant vitamins, antioxidant enzymes, and HSPs, may be altered in the presence of lower levels of oxidative stress and before the biomarkers of severe oxidative stress attributed to cytotoxicity appear. The alteration of early markers of oxidative stress, such as the oxidative stress-responsive gene HO-1 and plasma $\alpha$-tocopherol, without any increase in MDA or $8-\mathrm{OH}-$ $\mathrm{dG}$ after OTA, suggests that oxidative stress might be caused by some direct action of OTA (Gautier et al., 2001).

Low GSH concentrations in vitro (Schaaf et al., 2002; Kamp et al., 2005b) and in vivo have been reported after exposure to OTA (Gautier et al., 2001; Meki and Hussein, 2001; Ozcelik et al., 2004), as well as decreases in the activities of CAT, GPx, GR, and SOD in both liver and kidney (Meki and Hussein, 2001; Ozcelik et al., 2004). Here, we found that chronic treatment with OTA tended to reduce SOD mRNA levels, suggesting that OTA affects SOD at the transcriptional level too.

Several HSPs are induced by oxidative stress and/or exogenous chemical stressors at levels where no overt severe oxidative stress and cytotoxicity is observed (Beyersmann and Hechtenberg, 1997). Both HSP32 and HSP70 are active in the cellular defense mechanisms against stress injury. Normally, they act as molecular chaperones, performing important and well-known housekeeping functions. When cellular proteins become too oxidatively damaged to be repaired, chaperones switch their function from protein folding to protein digestion.

HSP32 was induced specifically in kidneys of OTAtreated rats (Gautier et al., 2001). No changes in the HSP70 level were detected in LLC-PK1 and MDCK cells treated with OTA (Barisic et al., 2002), and the relative distribution of two HSP70 isoforms (68- and 74$\mathrm{kDa}$ isoform) was affected by OTA, but with no change in the total amount of HSP70 in rat kidney (Barisic et al., 2002). No information about inducible HSP70 expression in rat liver is available to date. We observed significant down-regulation of HSP70 gene expression in rats after exposure to OTA, suggesting that OTA causes changes in critical proteins such as HSP70. Since the transient induction of HSP70 produces a state of increased resistance to further stress and the heat shock response is generally thought to be an adaptive mechanism to counteract adverse environmental conditions, the absence or strong reduction of HSP70 protective effects in hepatocytes might partly explain why the liver cannot counterbalance injury after exposure to OTA; this quite likely contributes at least partially to the mechanisms of OTA-induced liver toxicity in rats during long-term exposure.

Although OTA seems to induce mild oxidative stress and it was suggested that the antioxidant melatonin might protect against OTA toxicity in rat liver and kidney (Meki and Hussein, 2001; Ozcelik et al., 2004; Abdel-Wahhab et al., 2005), the absence of significant alterations in a number of late biomarkers of severe oxidative stress (e.g. protein carbonyls) after exposure to OTA (Kamp et al., 2005a; Mally et al., 2005a,b) seems to indicate that severe oxidative stress is not a major contributor to OTA cytotoxicity.

\subsection{OTA and tumor promotion}

Although OTA is a nephrotoxic and tumorigenic mycotoxin in experimental animals, we know little of the mechanisms of these effects. Since carcinogenesis consists of multiple stages and mechanisms, including mutagenesis, cytotoxicity and epigenetic alterations, it is important to clarify the potential mechanism by which OTA affects both rodent and human cells.

Previous studies (Zepnik et al., 2001) found that OTA did not exert a direct cytotoxic or genotoxic effect, since after its biotransformation by cytochrome $\mathrm{P} 450$ no reactive intermediates are formed capable of binding DNA. However, non-cytotoxic levels of OTA induced epigenetic mechanisms (Horvath et al., 2002), responsible in part for the tumor promotion phase of carcinogenesis.

Many tumor promoters affect gap junction intercellular communication (GJIC) and the expression of cadherins. GJIC plays a major role in maintaining tissue homeostasis and regulating cell growth, development and differentiation (Trosko and Ruch, 1998). Alterations in GJIC by toxicants is a recognized cellular 
event involved in carcinogenesis, particularly in the tumor-promoting process which facilitates clonal expansion of initiated cells by non-genotoxic mechanisms (Horvath et al., 2002; Yamasaki, 1990). The carcinogenic process involves the transition from a normal, GJICcompetent cell to one that is defective in GJIC, and most tumor promoters appear to inhibit GJIC in cultured cells (Budunova and Williams, 1994).

In adult liver, the main connexins are CX32 and CX26, which are expressed by adult parenchymal cells (hepatocytes). However, non-parenchymal liver epithelial cells, hepatic fat-storing cells, express CX43 (Trosko and Ruch, 1998). CX32, in particular, is the major gap junction protein expressed in hepatocytes, constantly expressed along the liver lobule (Spary et al., 1994). To our knowledge, this is the first study of rat liver GJIC after OTA treatment. OTA strongly reduced CX26, CX32 and CX43 gene expressions, suggesting that the toxin may exert a hepatotoxic effect as a non-genotoxic tumor promoter (Trosko et al., 1998). Similarly, hepatotoxicity induced by $\mathrm{CCl}_{4}$, which is thought to activate the inflammatory response and whose action is mediated by oxidative stress damage, reduced the expression of CX32 and CX26 (Saez et al., 1987). In the light of evidence of a major role of cell growth deregulation when GJIC is reduced in neoplastic and carcinogentreated cells, it can be proposed that chronic exposure to OTA may play a role in the promotion of hepatocarcinoma. Several studies have in fact suggested that CX32 expression had an inhibitory effect on hepatocarcinogenesis, and transfection with CX32 cDNA inhibits the growth of hepatoma cells (Eghbali et al., 1991; Evert et al., 2002). Interestingly, it was demonstrated a direct connection between oxidative stress and CX32 downregulation (Kojima et al., 1996). Furthermore, a modification of the binding of transcription factors to regulatory sequences in CX32 promotor was suggested as potential basis for this effect (Morsi et al., 2003). Accordingly, we can hypothesize that OTA-induced oxidative damage may affect $C X 32$ gene expression by a similar mechanism.

E-cadherin mediates cell-cell adhesion by association with intracellular catenins. E-cadherin is the main cadherin in the liver epithelium and its function may decline in the malignant progression of various tumors originating from epithelial cells, including liver tumors (Kanai et al., 1997; Gao et al., 2006). A reduction of E-cadherin, together with the down-regulation of DSC-2 and the tendency to reduced N-cadherin, therefore suggests that a loss of hepatocyte adhesive properties and a cell polarized phenotype may induce cell mobility, with a role in the mechanisms leading to the development of hepatocarcinoma (Mareel et al., 1997).

This idea is supported by our data showing a decrease in serum ALB and induction of AFP gene expression. The defective ALB production was reportedly due to the reduction or absence of $A L B$ gene transcription in some hepatoma cells. Interestingly, ALB production was increased in enhanced GJIC by $C X 32$ gene transfection (Yang et al., 2003).

\section{Conclusions}

These in vivo results show that the overall early effects of OTA on liver are different from those described in kidney, since the toxin did not exert a fibrogenic role (Gagliano et al., 2005). OTA-induced liver injury determines a weakening of the ability to counterbalance oxidative stress, and in particular altered GJIC and loss of cell adhesion and polarity. Since in vitro studies have shown that GJIC can be affected by oxidative stress and since alterations of GJIC and E-cad expression contribute to the promotion phase of tumorigenesis (Ruch and Klaunig, 1986; Gao et al., 2006), our findings strongly support the hypothesis that long-lasting OTA injury, such as mild oxidative damage in combination with other cytotoxic effects, might be the major determinant in the mechanisms leading to the development of hepatocarcinoma after a chronic exposure to OTA.

\section{Acknowledgments}

We thank Ms J.D. Baggott for editing the text. This work was supported by a grant of the Italian Government (Ministero delle Politiche Agricole e Forestali-MPAF) Targeted Project.

\section{References}

Abdel-Wahhab, M.A., Abdel-Gali, 1, M.M., El-Lithey, M., 2005. Melatonin counteracts oxidative stress in rats fed an ochratoxin A contaminated diet. J. Pineal Res. 38, 130-135.

Aydin, G., Ozcelik, N., Cicek, E., Soyoz, M., 2003. Histopathologic changes in liver and renal tissues induced by ochratoxin $\mathrm{A}$ and melatonin in rats. Hum. Exp. Toxicol. 22, 383-391.

Barisic, K., Petrik, J., Rumora, L., Cepelack, I., Grubisic, T.Z., 2002. Expression of Hsp70 in kidney cells exposed to ochratoxin A. Arch. Toxicol. 76, 218-226.

Baudrimont, I., Betbeder, A.M., Gharbi, A., Pfohl-Leszkowicz, A., Dirheimer, G., Creppy, E.E., 1994. Effect of superoxide dismutase and catalase on the nephrotoxicity induced by subchronical administration of ochratoxin A in rats. Toxicology 89, 101111. 
Beyersmann, D., Hechtenberg, S., 1997. Cadmium, gene regulation, and cellular signaling in mammalian cells. Toxicol. Appl. Pharmacol. 144, 247-261.

Budunova, I.V., Williams, G.N., 1994. Cell culture assays for chemicals with tumor-promoting or tumor-inhibiting activity based on the modulation of intercellular communication. Cell Biol. Toxicol. 10, 71-116.

Cassiman, D., Libbrecht, L., Desmet, V., Denef, C., Roskams, T., 2002. Hepatic stellate cell/myofibroblast subpopulations in fibrotic human and rat livers. J. Hepatol. 36, 200-209.

Dalle-Donne, I., Rossi, R., Giustarini, D., Gagliano, N., Di Simplicio, P., Colombo, R., Milzani, A., 2002. Methionine oxidation as a major cause of the functional impairment of oxidized actin. Free Radic. Biol. Med. 32, 927-937.

Dalle-Donne, I., Giustarini, D., Colombo, R., Rossi, R., Milzani, A., 2003a. Protein carbonylation in human diseases. Trends Mol. Med. 9, 169-176.

Dalle-Donne, I., Rossi, R., Giustarini, D., Milzani, A., Colombo, R., 2003b. Protein carbonyl groups as biomarkers of oxidative stress. Clin. Chim. Acta 329, 23-38.

Dalle-Donne, I., Rossi, R., Colombo, R., Giustarini, D., Milzani, A., 2006. Biomarkers of oxidative damage in human disease. Clin. Chem. 52, 601-623.

Eghbali, B., Kessler, J.A., Reid, L.M., Roy, C., Spray, D.C., 1991. Involvement of gap junctions in tumorigenesis: transfection of tumor cells with connexin $32 \mathrm{cDNA}$ retards growth in vivo. Proc. Natl. Acad. Sci. U.S.A. 88, 10701-10705.

Evert, M., Ott, T., Temme, A., Willecke, K., Dombrowski, F., 2002. Morphology and morphometric investigation of hepatocellular preneoplastic lesions and neoplasms in connexin32-deficient mice. Carcinogenesis 23, 697-703.

Gagliano, N., Torri, C., Donetti, E., Grizzi, F., Costa, F., Bertelli, A.A.E., Migliori, M., Filippi, C., Bedoni, M., Panichi, V., Giovannini, L., Gioia, M., 2005. Ochratoxin A-induced renal cortex fibrosis and epithelial-to-mesenchymal transition: molecular mechanisms of ochratoxin A-injury and potential effects of red wine. Mol. Med. 11, 30-38.

Gao, Z.-H., Tretiakova, M.S., Liu, W.-H., Gong, C., Farris, P.D., Hart, J., 2006. Association of E-cadherin, matrix metalloproteinases, and tissue inhibitors of metalloproteinases with the progression and metastasis of hepatocellular carcinoma. Modern Pathol. 19, 533-540.

Gautier, J.C., Holzhaeuser, D., Markovic, J., Gremaud, E., Schilter, B., Turesky, R.J., 2001. Oxidative damage and stress response from ochratoxin a exposure in rats. Free Radic. Biol. Med. 30, 1089-1098.

Halliwell, B., Whiteman, M., 2004. Measuring reactive species and oxidative damage in vivo and in cell culture: how should you do it and what do the results mean? Br. J. Pharmacol. 142, 231-255.

Horvath, A., Upham, B.L., Ganev, V., Trosko, J.E., 2002. Determination of the epigenetic effects of ochratoxin in a human kidney and a rat liver epithelial cell line. Toxicon 40, 273-282.

Kamp, H.G., Eisenbrand, G., Janzowski, C., Kiossev, J., Latendresse, J.R., Schlatter, J., Turesky, R.J., 2005a. Ochratoxin A induces oxidative DNA damage in liver and kidney after oral dosing to rats. Mol. Nutr. Food Res. 49, 1160-1167.

Kamp, H.G., Eisenbrand, G., Schlatter, J., Wurth, K., Janzowski, C., 2005b. Ochratoxin A: induction of (oxidative) DNA damage, cytotoxicity and apoptosis in mammalian cell lines and primary cells. Toxicology 206, 413-425.

Kanai, Y., Ushijima, S., Hui, A.M., Ochiai, A., Tsuda, H., Sakamoto, M., Hirohashi, S., 1997. The E-cadherin gene is silenced by CpG methylation in human hepatocellular carcinomas. Int. J. Cancer 71, 355-359.

Kojima, T., Mitaka, T., Mizuguchi, Y., 1996. Effects of oxygen radical scavengers on connexins 32 and 26 expression in primary cultures of adult rat hepatocytes. Carcinogenesis 17, 537-544.

Li, S., Marquardt, R.R., Frolich, A.A., 2000. Identification of ochratoxins and some of their metabolites in bile and urine of rats. Food Chem. Toxicol. 38, 141-152.

Mally, A., Volkel, W., Amberg, A., Kurz, M., Wanek, P., Eder, E., Hard, G., Dekant, W., 2005a. Functional, biochemical, and pathological effects of repeated administration of ochratoxin A to rats. Chem. Res. Toxicol. 18, 1242-1252.

Mally, A., Pepe, G., Ravoori, S., Fiore, M., Gupta, R., Dekant, W., Mosesso, P., 2005b. Ochratoxin A causes DNA damage and cytogenetic effects but no DNA adducts in rats. Chem. Res. Toxicol. $18,1253-1261$.

Mareel, M., Boterberg, T., Noe, V., Van Hoorde, L., Vermeulen, S., Bruynee, E., Bracke, M., 1997. E-cadherin/catenin/cytoskeleton complex: a regulator of cancer invasion. J. Cell. Physiol. 173, 271-274.

Meki, A.R., Hussein, A.A., 2001. Melatonin reduces oxidative stress induced by ochratoxin A in rat liver and kidney. Comp. Biochem. Physiol. C: Toxicol. Pharmacol. 130, 305-313.

Morsi, A.S., Godfrey, R.E., Chipman, J.K., Minchin, S.D., 2003. Characterisation of the connexin 32 promoter and changes in response element complexes in rat liver and hepatocytes during culture associated with oxidative stress. Toxicol. In Vitro 17, 191-199.

O'Brien, E., Dietrich, D.R., 2005. Ochratoxin A: the continuing enigma. Crit. Rev. Toxicol. 35, 33-60.

Ozcelik, N., Soyoz, M., Kilink, I., 2004. Effects of ochratoxin A on oxidative damage in rat kidney: protective role of melatonin. J. Appl. Toxicol. 24, 211-215.

Petkova-Bocharova, T., Chernozemski, I.N., Castegnaro, M., 1988. Ochratoxin A in human blood in relation to Balkan endemic nephropathy and urinary system tumours in Bulgaria. Food Addit. Contam. 5, 299-301.

Pfohl-Leszkowicz, A., Petkova-Bocharova, T., Chermozemsky, I.N., Castegnaro, M., 2002. Balkan endemic nephropathy and associated urinary tract tumours: a review on aetiological causes and the potential role of mycotoxins. Food Addit Contam 19, 282302.

Petzinger, E., Ziegler, K., 2000. Ochratoxin A from a toxicological perspective. J. Vet. Pharmacol. Ther. 23, 91-98.

Ruch, R.J., Klaunig, J.E., 1986. Antioxidant prevention of tumor promoter induced inhibition of mouse hepatocyte intercellular communication. Cancer Lett. 33, 137-150.

Saez, J.C., Bennett, M.V., Spay, D.C., 1987. Carbon tetrachloride at hepatotoxic levels blocks reversibly gap junctions between rat hepatocytes. Science 236, 967-969.

Schaaf, G.J., Nijmaijer, S.M., Maas, R.F.M., Roestenberg, P., de Groene, E.M., Fink-Gremmels, J., 2002. The role of oxidative stress in the ochratoxin A-mediated toxicity in the proximal tubular cells. Biochim. Biophys. Acta 1588, 149-158.

Spary, D.C., Bai, S., Burk, R.D., Saez, J.C., 1994. Regulation and function of liver gap junctions and their genes. Prog. Liver Dis. 12, $1-18$.

Trosko, J.E., Ruch, R.J., 1998. Cell-cell communication in carcinogenesis. Front. Biosci. 3, d208-d236.

Trosko, J.E., Chang, C.C., Upham, B., Wilson, M., 1998. Epigenetic toxicology as toxicant-induced changes in intracellular signalling leading to altered gap junctional intercellular communication. Toxicol. Lett. 102/103, 71-78. 
Vrabcheva, T., Petkova-Bocharova, T., Grosso, F., Nikolov, I., Chernozemsky, I.M., Castegnaro, M., Dragacci, S., 2004. Analysis of ochratoxin A in foods consumed by inhabitants from an area with Balkan endemic nephropathy: a 1-month follow-up study. J. Agric. Food Chem. 52, 2404-2410.

Yamasaki, H., 1990. Gap junctional intercellular communication and carcinogenesis. Carcinogenesis 11, 1051-1058.
Yang, J., Ichikawa, A., Tsuchiya, T., 2003. A novel function of connexin 32: marked enhancement of liver function in a hepatoma cell line. Biochem. Biophys. Res. Commun. 307, 80-85.

Zepnik, H., Pahler, A., Schauer, U., Dekant, W., 2001. Ochratoxin Ainduced tumor formation: is there a role of reactive ochratoxin $\mathrm{A}$ metabolites? Toxicol. Sci. 59, 59-67. 